\title{
Validación Experimental de la Tolerancia a Fallas en un Hexa-rotor con Brazos Inclinados
}

\section{Experimental Validation of Fault Tolerance in a Hexa-rotor with Inclined Arms}

\section{Juan I. Giribet}

Grupo de Procesamiento de Seliales, Identificación y Control. Departamento de Ingeniera Electrónica, Universidad de Buenos Aires y CONICET - Buenos Aires - Argentina

jgiribet@fi.uba.ar

\section{Claudio Pose}

Grupo de Procesamiento de Seliales, Identificación y Control. Departamento de Ingeniera Electrónica, Universidad de Buenos Aires - Buenos Aires - Argentina

cldpose @ fi.uba.ar

\section{Ignacio Mas}

Centro de Sistemas y Control, Departamento de Matemática, Instituto Tecnológico de Buenos Aires y CONICET - Buenos Aires - Argentina

imas@itba.edu.ar 


\title{
Resumen
}

Recientemente, han surgido algunos trabajos re-portando las ventajas de volar con multicópteros con rotores inclinados, es decir, rotores que no apunten en la dirección de la vertical del vehículo. En particular, se demostró que para lograr un vehículo hexa-rotor capaz de tolerar fallas en uno de sus rotores es necesario que estos estén inclinados. Hasta el momento, las validaciones experimentales de estos resultados han considerado fallas parciales en los rotores. Más precisamente, se han analizado casos en los cuales uno de los rotores pierde capacidad de empuje. Sin embargo, los resultados teóricos admiten una falla total de los rotores, es decir, que uno de los rotores se detenga completamente. En este trabajo validamos experimentalnente la falla total de un rotor y mostramos como un vehículo hexa-rotor es capaz de mantener su capacidad de maniobra ante la pérdida total de uno de sus rotores.

Palabras Claves: Vehículos aéreos no tripulados, vehículos multi_rotores, tolerancia a fallas.

\begin{abstract}
Recently, some work has emerged re-carrying the advantages of flying with multicopters with inclined rotors, that is, rotors that do not point in the direction of the vertical of the vehicle. In particular, it was shown that in order to achieve a hexa-rotor vehicle capable of tolerating faults in one of its rotors, it is necessary that they be inclined. So far, experimental validations of these results have considered partial failures in the rotors. More precisely, cases have been analyzed in which one of the rotors loses thrust capacity. However, the theoretical results admit a total failure of the rotors, that is, one of the rotors stops completely. In this work we experimentally validate the total failure of a rotor and show how a hexa-rotor vehicle is capable of maintaining its maneuverability in the face of the total loss of one of its rotors.
\end{abstract}

Keywords: Unmanned aerial vehicles, multi-engine vehicles, fault tolerance.

\section{Introducción}

En los últimos años los avances en diversos campos como la ingeniería electrónica, mecánica e informática han im-pulsado el desarrollo de los vehículos aéreos no tripulados (VANT). Estos vehículos, tanto aquellos tele-operados, como los más avanzados de navegación autónoma, están empezando a reemplazar a los sistemas tripulados en diversos ambientes de operación, teniendo beneficios en lo que respecta a la capacidad de repetición de tareas, reducción de los riesgos para los pilotos u operación en entornos peligrosos como incendios, catástrofes naturales o industriales, por ejemplo en plantas químicas o centrales nucleares.

Si bien los VANT han sido utilizados desde hace varias décadas, en los últimos años han adquirido gran popularidad. Esto ha impulsado el surgimiento de nuevas empresas que han crecido de manera notable. Muchas de estas empresas comer $\neg$ cializan sus VANT para el 
público en general, algunos de estos vehículos incluso vienen equipados con cámaras y son capaces de volar de manera autónoma. Sin embargo, como sucede con muchas tecnologías, su use indebido puede ser riesgoso. En los últimos años diversos accidentes provocados por drones han sido reportados. Por otro lado, también han aparecido en di-versos medios de comunicación casos de VANT volando sobre lugares indebidos como aeropuertos, espectáculos deportivos, manifestaciones y diversas situaciones en las cuales podría producirse un accidente en caso que se presentara una falla en el vehículo.

\section{Clasificaciones y características de los vehículos aéreos no tripulados}

Existen diversos tipos de VANT, de ala fija, helicópteros (con rotor de cola, autogiros, multicópteros o multi-rotores) y otros menos frecuentes como globos o zeppelins. Algunos de estos vehículos dependen completamente de los comandos de un piloto en tierra, mientras que otros son capaces de tomar decisiones de manera autónoma, como decidir abortar una misión en caso de considerarla riesgosa, por ejemplo si evalúa que la batería no tiene la carga suficiente para finalizar la misión. También el vehículo podría tomar la decisión de evitar determinada zona por considerarla peligrosa, por ejemplo porque está en las cercanías de un aeropuerto, entre otras capacidades.

Cada uno de estos vehículos tiene sus ventajas, para ciertas aplicaciones la capacidad de maniobra de los helicópteros los hace una plataforma más adecuada que los aviones. Por ejemplo, para filmación aérea de eventos, comerciales, etc., las tomas desde el aire en forma estática no pueden realizarse con un avión. Son \&Hes también para realizar la inspección de obras civiles, torres de alta tensión, de antenas, entre tantas otras aplicaciones. Los helicópteros también pueden ser utili־zados en aplicaciones que requieran que el VANT se desplace de un punto a otro, al igual que los VANT de ala fija. Es cierto que, estos últimos son más adecuados para aplicaciones en las cuales se necesario recorrer grandes distancias, ya que tienen mayor autonomía de vuelo. Sin embargo los helicópteros son más versátiles desde el punto de vista de la capacidad de maniobras, por lo cual son una plataforma muy atractiva y necesaria. En particular los helicópteros del tipo multi-rotor tienen una mecánica muy simple, a diferencia de un helicóptero convencional con rotor de cola, no requieren partes mecánicas móviles. Su desventaja es que por lo general tienen menor autonomía de vuelo, debido a que son eléctricos y las baterías permiten una autonomía de vuelo de 15 a 40 minutos, dependiendo del tamaño del vehículo y la carga útil. Pese a esta limitación, los multicópteros eléctricos son uno de los VANT que han tornado mayor notoriedad en los últimos arios. Son simples de reparar, construir, calibrar y son vehículos más fáciles de controlar. Por otro lado, pueden lograrse diseños tolerantes a fallas, que es un requerimiento fundamental que nunca debe dejarse de lado.

De esta forma si se desea un vehículo versátil, capaz de ejecutar diversas maniobras y volar en ambientes cerrados, con una mecánica simple y capaz de tolerar fallas en alguno de sus rotores, un vehículo multi-rotor eléctrico se presenta como la mejor alternativa.

Existe una amplia gama de vehículos del tipo multi-rotor, usualmente con tres, cuatro, seis u ocho rotores. Los vehículos de tres rotores no son completamente controlables en orientación, esto significa que existe un torque que no son capaces de lograr, por ejemplo no es posible lograr un movimiento en pitch sin que este provoque un movimiento en yaw (ver figura 1), por otro lado los vehículos de cuatro o más rotores si son completamente controlables en orientación, es decir poseen 3 grados de libertad (3DOF) en orientación. Más aun, son vehículos con 4DOF debido a que (en torno a su punto nominal de operaci6n) pueden variar su altura indepen $\neg$ dientemente de las variaciones en orientación. Sin embargo, no Este trabajo ha sido financiado por la Agencia Nacional de Promoción Científica y Tecnológica, FONCYT PICT 2014-2015 (Argentina). Claudio Pose agradece a la fundación Peruilh y a la Facultad de Ingeniera de la Universidad de Buenos Aires por su apoyo. 
son completamente controlables en orientación y posición puesto que para provocar un movimiento en el eje x o y es necesario variar el pitch o roll del vehículo, respectivamente. Sin embargo, es posible lograr un vehículo con 6DOF, es decir capaz de moverse en orientación y en cualquier dirección de manera independiente, pero para esto es necesario torcer los rotores respecto de la vertical del vehículo, que es como usualmente se ubican para maximizar el empuje vertical. En un vehículo de seis o más rotores, si estos se tuercen un Angulo pequeño respecto de la vertical (para no perder mucho empuje en el eje z) es posible lograr que el vehículo se mueva en cualquier dirección y con cualquier orientación [1] - [3]. Pero estas configuraciones no son usuales y por lo general se considera que los multi-rotores son vehículos con 4DOF.

Este trabajo se centra en el problema de tolerancia a fallas en los rotores. Más precisamente, se desea lograr un vehículo que, aun si uno de sus rotores dejara de funcionar sea capaz de mantener el control en cualquiera de sus ángulos, controlando la altura (es decir un vehículo con 4DOF). No solo se pretende un vehículo que permanezca en el aire aun si un rotor falla, se desea un vehículo que no pierda capacidad de maniobrar, lo cual aporta mayor seguridad.

La primera pregunta que surge es cuantos rotores debe tener un vehículo para tener esta propiedad. Es sabido que para mantener en vuelo un vehículo basta con un rotor [4], pero son necesarios al menos cuatro rotores para lograr 4DOF. En [3], [5] se probó que seis es la mínima cantidad de rotores necesarios para lograr un vehículo tolerante a fallas. Más aun, se demostró que el diseño utilizado usualmente no es adecuado

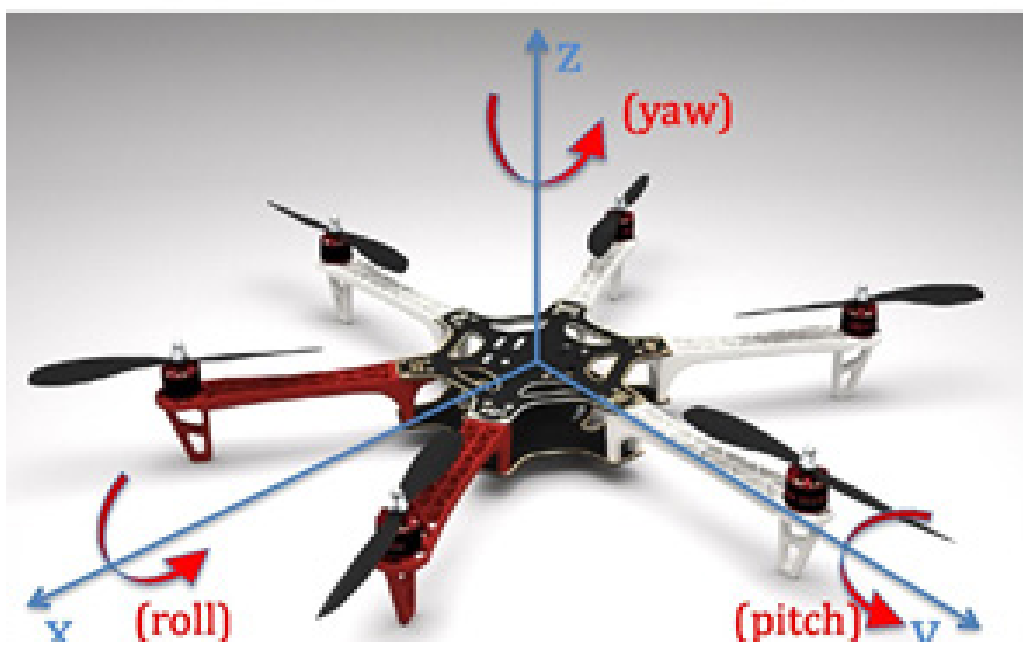

Figura 1. Ángulos y ejes del multicoptero.

para este propósito. Recientemente han sido propuestos nuevos diseños para los vehículos de seis rotores que si logran la tolerancia a fallas, es decir que son capaces de mantener 4DOF aun si uno de sus rotores falla, suponiendo que la falla puede presentarse en cualquiera de sus rotores. De estos diseños, el que resulta más simple es el propuesto en [3] debido a que requiere tan solo que los rotores (o los brazos del vehículo) estén inclinados cierto ángulo respecto de la vertical del vehículo.

Los resultados publicados en [3] son teóricos y no presentan resultados experimentales. Recientemente, en [6], [7] se han validado estos resultados mediante simulaciones y si bien 
se indica que se han llevado a cabo resultados experimentales que validan la técnica, estos no han sido incluidos en los trabajos. Por otro lado, en [8] han sido reportados resultados experimentales en donde se provoca una falla en uno de los rotores del vehículo, pero esta falla es parcial, provocando una pérdida del empuje del rotor, aunque este sigue funcionando. El propósito de este trabajo es validar experimentalmente los resultados propuestos en [3], mostrando como un vehículo de seis rotores puede mantener sus 4DOF aun ante la pérdida total de uno de sus rotores.

\section{MODELO DEL VEHÍCULO MULTI-ROTOR}

Cada rotor (esto significa, cada conjunto motor/hélice) es capaz de ejercer una fuerza en una dirección (es decir $\mathrm{f}_{\mathrm{i}}>0$ ), debido a que por lo general los rotores son capaces de girar en un solo sentido y la hélice se construye de forma tal que permita el empuje en la dirección adecuada. En la práctica la computadora de a bordo del vehículo comanda la fuerza de cada rotor $f_{i}$, enviando una serial $\mathrm{u}_{i}$ (Pulse Width Modulated - PWM) con valores entre 0 y $100 \%$, la cual nominalmente puede considerarse proporcional a la fuerza que ejerce el motor $\mathrm{f}_{\mathrm{i}}=k f u_{i}$, donde $k f$ es una constante que depende de las características del rotor del vehículo.

Por otro lado, cada rotor ejerce un torque sobre su eje de rotación, $m_{i}=(-1)$ iktui. La constante kt es otro parámetro que depende de las características mecánicas del rotor, mien $\neg$ tras que el signo (-1) i depende de si el i-esimo rotor gira en sentido horario o anti-horario. Es necesario contar con rotores

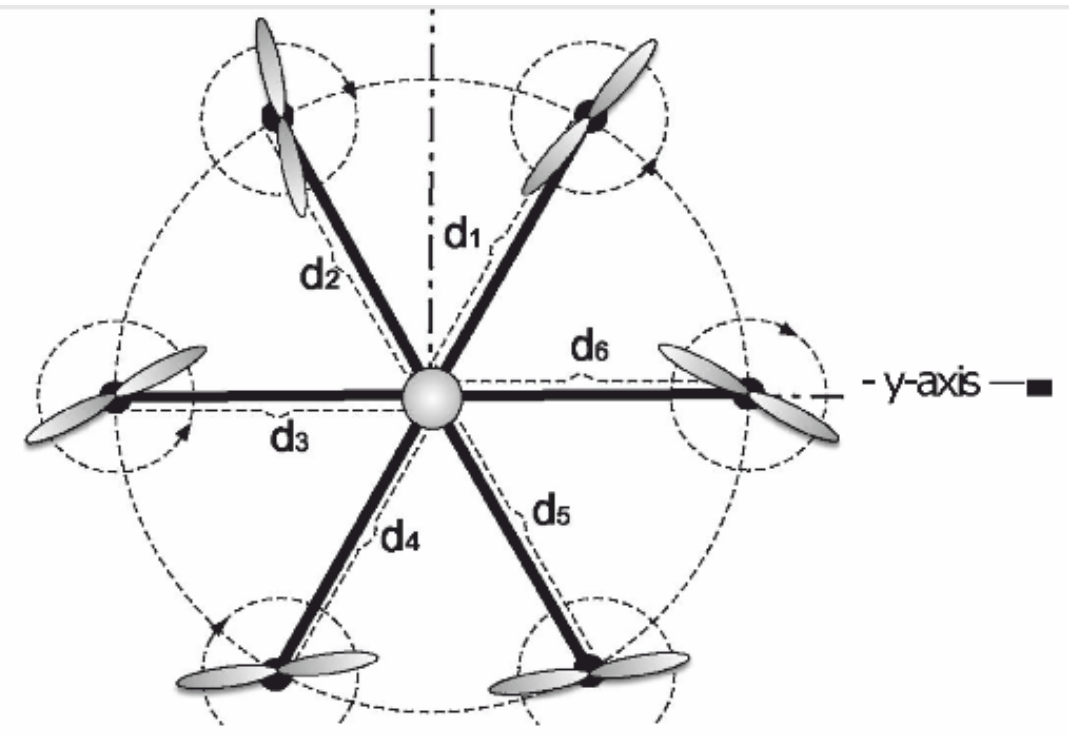

Figura 2. Configuración de un vehículo de 6 rotores (Vista de abajo).

que giren en ambos sentidos, para poder compensar el torque en el eje z.

Las Figuras 2 y 3 muestran la disposición de los rotores para un vehículo hexa-rotor. El empuje total $F_{z}$ del vehículo es en la dirección del eje z, y será positivo $\left(F_{z}>0\right)$. Si se desea maximizar el empuje, el ángulo $\gamma$ (ver Figura 3) debe elegirse $\gamma=90$ grados, que es el 
desafío estándar de los vehículos de seis rotores. Sin embargo, como se verá más adelante para esta configuración el vehículo no es capaz de lograr 3DOF en orientación si se pierde uno de sus rotores.

En adelante, para simplificar la notación, se define el parámetro:

$$
\alpha=\alpha(\gamma)=\frac{k_{t} \cos (\gamma)}{k_{f} \sqrt{3} l \sin (\gamma)},
$$

donde 1 es la longitud de los brazos del vehículo (que se suponen todas iguales). Se considera que 7 es un ángulo estrictamente mayor que 0 y menor que 180 grados (de lo contrario no se podría ejercer empuje en la dirección z). Cabe remarcar que el parámetro a, en la configuración estándar (es decir, con $\gamma=90$ grados) resulta $\mathrm{a}=0$.

Por otro lado, si se desea realizar una maniobra de rotación con el vehículo, es necesario que los rotores ejerzan un torque $\mathrm{M}=\left(\mathrm{M}_{\mathrm{x}}, \mathrm{M}_{\mathrm{y}}, \mathrm{M}_{\mathrm{z}}\right) \mathrm{E} \mathrm{R}^{3}$ en cada uno de los ejes, dependiendo de la direcci6n en la que se desea rotar. Como se mencionó anteriormente, es deseable que el vehículo sea capaz de maniobrar en cualquier dirección. La magnitud del momento deseado estará limitada por la fuerza máxima que

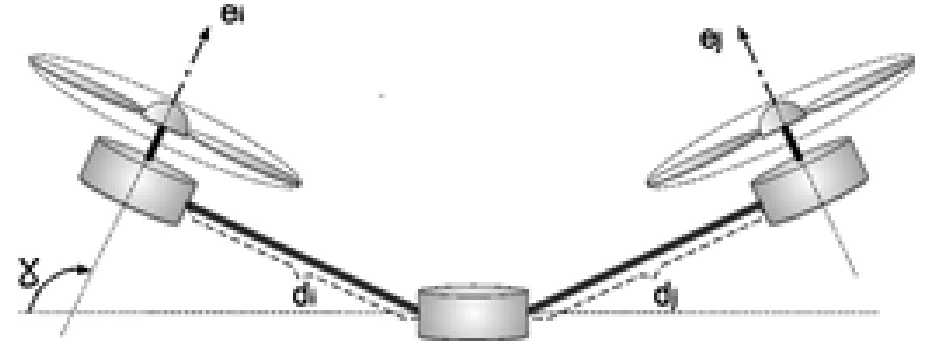

Figura 3. Configuración de un vehículo de 6 rotores (Vista de costado).

puedan alcanzar los rotores, pero se desea que la dirección del vector de momentos $\mathrm{M} \varepsilon$ R3 pueda ser arbitraria.

La relación de la fuerza alcanzada por cada uno de los rotores $f_{2}>0$ con el torque y empuje $\left(M_{x^{\prime}}, M_{y}, M_{z}, F_{z}\right)$ realizado sobre el vehículo está dada por la siguiente ecuación:

$$
\left[\begin{array}{c}
M_{x} \\
M_{y} \\
M_{z} \\
F_{z}
\end{array}\right]=A(\gamma, \alpha) \cdot f, \quad \text { con } \quad f=\left[\begin{array}{c}
f_{1} \\
\vdots \\
f_{6}
\end{array}\right],
$$

donde la matriz $A=A(\gamma, \alpha) \in \mathbb{R}^{4 \times 6}$ es:

$$
\left[\begin{array}{cccccccc}
\frac{k_{t}}{k f} c(\gamma) & {[} & -1 & \frac{\alpha+1}{2 \alpha} & \frac{\alpha+1}{2 \alpha} & -1 & \frac{\alpha-1}{2 \alpha} & \left.\frac{\alpha-1}{2 \alpha}\right] \\
\frac{k t}{k f} c(\gamma) & {[} & -\frac{1}{\sqrt{3} \alpha} & \frac{3 \alpha-1}{2 \sqrt{3} \alpha} & -\frac{3 \alpha-1}{2 \sqrt{3} \alpha} & \frac{1}{\sqrt{3} \alpha} & \frac{3 \alpha+1}{2 \sqrt{3} \alpha} & \left.-\frac{3 \alpha+1}{2 \sqrt{3} \alpha}\right] \\
\frac{k_{t}}{k f} s(\gamma) & {[} & 1 & -1 & 1 & -1 & 1 & -1] \\
s(\gamma) & {[} & 1 & 1 & 1 & 1 & 1 & 1]
\end{array}\right] .
$$

Cabe aclarar que, teniendo en cuenta que

$$
k_{t} \cos (\gamma) / \alpha=k_{f} \sqrt{(3)} l \sin (\gamma),
$$


la matriz A $(\gamma, \alpha)$ está bien definida para todo valor de a.

De esta manera, el problema de hallar la fuerza $f_{i}>0$ de cada uno de los rotores para lograr el torque $\mathrm{M} \varepsilon \mathrm{R}^{3}$ y empuje $\mathrm{F}_{\mathrm{z}}>0$ deseado, se resuelve encontrando las soluciones de (2). Usualmente este problema se resuelve utilizando la pseudoinversa de Moore-Penrose de A, denotada At (ver por ejemplo [9]), y así las soluciones se obtienen mediante:

$$
\left[\begin{array}{c}
f_{1} \\
\vdots \\
f_{6}
\end{array}\right]=A(\gamma, \alpha)^{\dagger}\left[\begin{array}{c}
M_{x} \\
M_{y} \\
M_{z} \\
F_{z}
\end{array}\right]+w .
$$

donde $w \in N(A)$, es un vector en el núcleo de $A=A(\gamma, \alpha)$.

Usualmente, se toma $\mathrm{w}=0 \mathrm{y}$ así la solución está dada por la pseudoinversa de MoorePenrose A $(y, a)$ t, esto se debe a que de esta manera se obtiene la solución de mínima norma eucli $\neg$ dea (que resulta la de energía mínima) del problema (2). Sin embargo, cabe aclarar que son necesarias hipótesis adicionales para garantizar que la solución dada por la pseudoinversa de Moore-Penrose satisfaga $f_{i}>0$ para todo $i=1, \ldots, 6$, ver por ejemplo [3], [7].

Es el estudio de las soluciones de un problema como el dado por la ecuación (2) lo que permite analizar la capacidad del vehículo para ser (o no) tolerante a fallas. Para estudiar este problema se debe analizar cómo se modela una falla en uno de los rotores.

11-A. Falla en los rotores y análisis de la tolerancia a fallas

Se supondrá que las fallas en los rotores son totales, esto significa que si un rotor falla, no es capaz de generar fuerza. De esta manera, por ejemplo, si el rotor 2 presenta una falla, entonces $12=0$. Así, si se desea lograr un torque $(\mathrm{Mx} \mathrm{My}, \mathrm{Mz}) \mathrm{E} \mathrm{R}^{3}$ y un empuje $F_{z}>0$, entonces se desea estudiar si existen soluciones de la ecuación (2), con $12=0$.

Otra forma de modelar la falla del rotor 2 seria reemplazar la columna 2 de la matriz A por una columna con ceros. Se define la matriz $A_{i} \varepsilon R^{4 \times 6}$ como aquella que se obtiene

reemplazando la columna i de la matriz A por ceros. De esta manera, para una falla del rotor 2 se define A2 como: 


$$
\left[\begin{array}{cccccccc}
\frac{k t}{k f} c(\gamma) & {[} & -1 & 0 & \frac{\alpha+1}{2 \alpha} & -1 & \frac{\alpha-1}{2 \alpha} & \left.\frac{\alpha-1}{2 \alpha}\right] \\
\frac{k_{t}}{k f} c(\gamma) & {[} & -\frac{1}{\sqrt{3} \alpha} & 0 & -\frac{3 \alpha-1}{2 \sqrt{3} \alpha} & \frac{1}{\sqrt{3} \alpha} & \frac{3 \alpha+1}{2 \sqrt{3} \alpha} & \left.-\frac{3 \alpha+1}{2 \sqrt{3} \alpha}\right] \\
\frac{k_{t}}{k f} s(\gamma) & {[} & 1 & 0 & 1 & -1 & 1 & -1] \\
s(\gamma) & {[} & 1 & 0 & 1 & 1 & 1 & 1]
\end{array}\right] .
$$

Ante una falla del rotor 2 , si se desea lograr un torque $\left(M_{x}, M_{y}, M_{z}\right)$ y un empuje $F_{z}$, las fuerzas de los rotores $\left(f_{i} \geq 0\right.$ y $\left.f_{2}=0\right)$ están dadas por:

$$
\left[\begin{array}{c}
f_{1} \\
\vdots \\
f_{6}
\end{array}\right]=A_{2}(\gamma, \alpha)^{\dagger}\left[\begin{array}{c}
M_{x} \\
M_{y} \\
M_{z} \\
F_{z}
\end{array}\right]+\beta\left(\begin{array}{c}
\frac{3}{4}(\alpha-1)\left(\alpha+\frac{1}{3}\right) \\
0 \\
-\frac{3}{4}(\alpha+1)\left(\alpha-\frac{1}{3}\right) \\
-\frac{3}{4}\left(\alpha^{2}+\frac{1}{3}\right) \\
\alpha \\
\frac{3}{4}\left(\alpha^{2}+\frac{1}{3}\right)
\end{array}\right)
$$

donde $A_{2}^{\dagger}$ está dada por la ecuación (5), el vectro $w=$

$$
\frac{3}{4}\left((\alpha-1)\left(\alpha+\frac{1}{3}\right), 0,-(\alpha+1)\left(\alpha-\frac{1}{3}\right),-\left(\alpha^{2}+\frac{1}{3}\right), \frac{4}{3} \alpha,\left(\alpha^{2}+\frac{1}{3}\right)\right)^{T}
$$

es un vector en $\mathrm{N}$ (A2) que satisface la restricción $\mathrm{w} 2=0$. Mas min, todos los vectores en $N\left(A_{2}\right)$ que satisfacen esta restricción, se escriben de la forma $\beta \omega$, con $\beta € R$ parametri-zando el conjunto de todas las soluciones de la ecuación (4) con la restricción $1_{2}=0$.

Para analizar por qué el desafío estándar para un hexa-rotor no es tolerante a fallas y si lo es cuando se inclinan los mo $\neg$ tores, basta con analizar la última columna de la matriz $\mathrm{Al}^{t}$. Es fácil ver que los elementos de esta resultan estrictamente positivos, siempre que a $\neq 0 \mathrm{Y} \mathrm{IaI} \neq$ 1. Esto hace que siempre sea posible lograr un torque en cualquier dirección. De hecho, si se desea lograr el torque $(\mathrm{Mx}, \mathrm{My}, \mathrm{Mz}) \varepsilon \mathrm{R}^{3}$, alcanza con elegir $F_{z}>0$ suficientemente grande de manera tal que $f_{i}>0$ (notar que de la ecuación (4), siempre resulta $f_{i}=0$, que corresponde al motor fallado). Sin embargo, si a $=0$ o al $=1$ esto no es posible. Por ejemplo, si a $=0$, es decir si los rotores se ubican apuntando en la dirección z, resulta que la fuerza del rotor 5 (es decir, el rotor opuesto al que falla) está dada por:

$$
f_{5}=\left(\frac{\alpha\left(3 \alpha^{3}-1\right) \sec (\gamma)}{k_{t}\left(3 \alpha^{2}+1\right)^{2}}\right) M_{x}+\left(\frac{\alpha\left(9 \alpha^{3}+1\right) \sec (\gamma)}{\sqrt{3} k_{t}\left(3 \alpha^{2}+1\right)^{2}}\right) M_{y}+\left(\frac{\csc (\gamma)}{9 k_{t} \alpha^{2}+3 k_{t}}\right) M_{z},
$$

la cual no depende de $F_{z}$ ni de $\beta$. Por lo tanto existe un momento $M=\left(M_{x}, M_{y}, M z\right)$ para el cual no existen soluciones positivas $f_{i} \geq 0$ de la ecuación (4). Por ejemplo, si $\alpha=0$, la maniobra correspondiente al torque

$$
M=-\varepsilon\left(\frac{\alpha\left(3 \alpha^{3}-1\right) \sec (\gamma)}{k_{t}\left(3 \alpha^{2}+1\right)^{2}}, \frac{\alpha\left(9 \alpha^{3}+1\right) \sec (\gamma)}{\sqrt{3} k_{t}\left(3 \alpha^{2}+1\right)^{2}}, \frac{\csc (\gamma)}{9 k_{t} \alpha^{2}+3 k_{t}}\right)
$$

no puede ser ejecutada por el vehículo, para ningún valor de $\varepsilon>0$.

Lo mismo ocurre, si $|\alpha|=1$. Esto significa que, si los rotores se ubican en un ángulo $\gamma=90$ grados, o tal que 


\begin{abstract}
$|\tan (\gamma)|=\frac{k_{t}}{k_{f} \sqrt{3} i}$, entonces el vehículo no será capaz de lograr algunas maniobras, perdiendo grados de libertad para controlar el vehículo. Sin embargo, si se ubican los rotores en cualquier otro valor de $\gamma$, entonces es posible lograr que el vehículo ejecute cualquier maniobra y así obtener un vehículo con $4 \mathrm{DOF}$. La pregunta que resta resolver es cómo conviene elegir el valor de $\gamma$.
\end{abstract}

\title{
DISEÑO PROPUESTO PARA UN VEHÍCULO TOLERANTE A
}

\section{FALLAS}

Resulta evidente que, con el propósito de maximizar el empuje, es conveniente elegir $\gamma=$ 90 grados, es decir $a=0$. Por otro lado, para no perder la capacidad de tolerar fallas se debe cumplir con $\alpha \neq 0$.

Dependiendo del ángulo $\gamma$ elegido, y dada una cota para la magnitud del empuje ejercido, existe una dirección que es la más difícil de lograr (que exige más a los rotores), este torque se denomina el torque de peor caso. La Figura 4, muestra (para un vehículo con constantes kt,kf,1 fijas) el empuje Fz necesario para lograr el torque de peor caso para distintos valores del ángulo $9=180-\gamma$. En esta figura puede apreciarse

$$
\text { que, cuando } \bar{\gamma}=90 \text { grados o cuando }|\tan (\gamma)| \stackrel{\cdot}{=} \frac{k_{1}}{k_{f} \sqrt{3} l}
$$

(que corresponde a $\gamma=45$ grados y $7=135$ grados, aproximadamente) se tiene que $F_{z} \rightarrow$ $\infty$, debido a que existe una dirección del torque que no es posible alcanzar. Por otro lado, si $\gamma=73$ grados o $\gamma=107$ grados, el empuje necesario para rechazar el torque de peor caso se minimiza. Es posible ver además que si los rotores se ubican en estos ángulos, se pierde menos del $5 \%$ del empuje del vehículo. Dado que está perdida de empuje no se considera significativa, en el valor que se realiza el mínimo se puede fijar el valor del ángulo de inclinación para los rotores.

En la Figura 5 puede observarse un hexa-rotor volando con una falla en uno de sus rotores. Este vehículo es un modelo comercial con modificaciones que se han llevado a cabo en el Grupo de Procesamiento de Señales, Identificación y Control (GPISC) de la Facultad de Ingeniería de la Universidad de Buenos Aires [10]. La computadora de vuelo, así como los

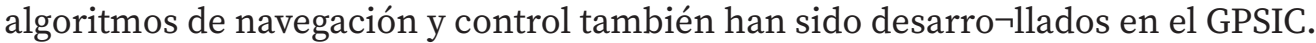

Desde el punto de vista de la tolerancia a fallas hay cierta simetría en la elección de $\gamma=107$ grados o $\gamma=73$ grados. Sin embargo, hay otros factores que hacen que $\gamma=107$ grados sea más conveniente. El primer motivo se basa en una cuestión de estabilidad de vuelo del vehículo y el segundo factor tiene que ver con que estos vehículos suelen utilizar cámaras instaladas en la parte de abajo. Inclinar los brazos hacia arriba permite mejorar el campo de visión de la cámara, motivo por el cual algunos vehículos comerciales vienen con los brazos inclinados. 


\section{RESULTADOS EXPERIMENTALES}

El propósito de esta sección es mostrar los resultados experimentales obtenidos en un vuelo del vehículo hexa-rotor. Se presentan resultados del vehículo operando con y sin falla. No se tratará aquí el problema de detección de falla ni la conmutación del control de un estado nominal a un estado de falla. El propósito es tan solo demostrar experimentalmente que el vehículo es capaz de volar con un motor menos y ejecutar diversas maniobras.
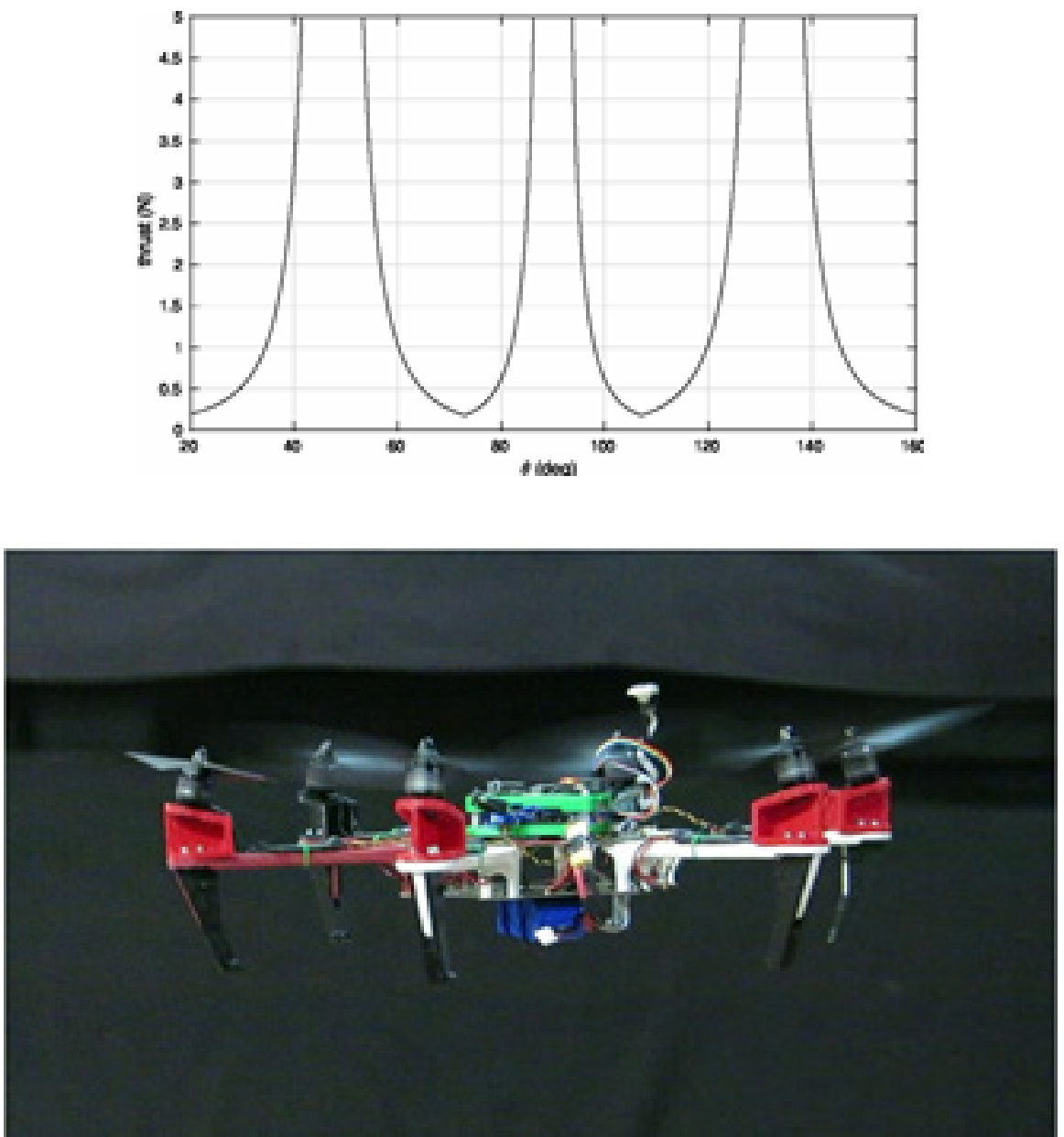

Figura 5. Vehículo multi-rotor volando con una falla en uno de sus rotores.

A continuación se muestra el experimento realizado para un vehículo modelo DJI F550, motores de $920 \mathrm{KV}$ con hélices 9545 y batería LiPo 4s.

Los datos obtenidos durante el vuelo corresponden a las Figuras 6 y 7. Durante los primeros 35 segundos se realiza un vuelo normal sin falla para demostrar las capacidades de vuelo del vehículo. Puede notarse en la Figura 6, donde se observan los ángulos de pitch, roll y yaw, que el error respecto de las referencias en los primeros dos ángulos es menor a $0.5^{\circ}$ durante el hovering; así como también es notable el buen desempeño del vehículo al 
momento de seguir las referencias comandadas en cualquier dirección. En la Figura 7 se grafican los valores de PWM entre 0 y $100 \%$ de cada uno de los motores, los cuales, como es de esperar, funcionan todos aproximadamente a la misma velocidad, producto de la simetría del vehículo.

$$
A_{2}(\gamma, \alpha)^{\dagger}=\left[\begin{array}{c}
-\frac{\alpha\left(15 \alpha^{3}+9 \alpha^{2}+9 \alpha-1\right) \sec (\gamma)}{4 k_{t}\left(3 \alpha^{2}+1\right)^{2}} \\
0 \\
\frac{3 \alpha(\alpha+1)^{3} \sec (\gamma)}{4 k_{t}\left(3 \alpha^{2}+1\right)^{2}} \\
-\frac{\alpha(3 \alpha-1) \sec (\gamma)}{4\left(3 k_{t} \alpha^{2}+k_{t}\right)} \\
\frac{\alpha\left(3 \alpha^{3}-1\right) \sec (\gamma)}{k_{t}\left(3 \alpha^{2}+1\right)^{2}} \\
\frac{\alpha(3 \alpha-1) \sec (\gamma)}{4\left(3 k_{t} \alpha^{2}+k_{t}\right)}
\end{array}\right.
$$
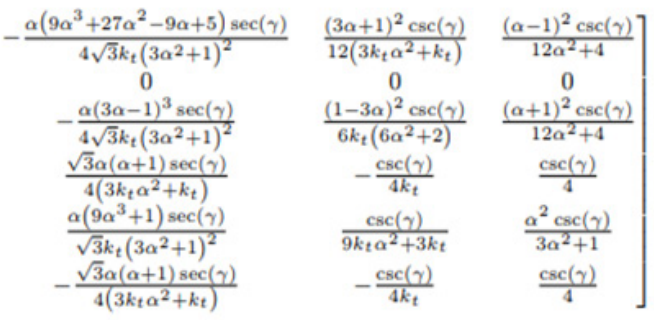

A partir de los 35 segundos, el vehículo aterriza y vuelve a despegar, esta vez con una falla total en el motor 2 que lo mantiene apagado durante todo el vuelo. Dado que en esta situación el sistema de control del vehículo está fuerᄀtemente degradado, debido a las nuevas limitaciones para ejercer torques en cualquier dirección, es de esperar que el desempeño durante el vuelo sea menor, como puede observarse en la Figura 6 durante la parte intermedia del vuelo, en hovering, con ligeras oscilaciones en torno a los $3^{\circ}$. Las fuertes oscilaciones durante los primeros y últimos segundos del vuelo con falla se deben a la cercanía al piso en un despegue y aterrizaje lento, donde se produce un fuerte efecto suelo generando grandes perturbaciones de forma despareja, dado que bajo el motor apagado no se produce dicho efecto. Mientras se realiza el vuelo con falla, puede apreciarse ahora entre los 35 y 70 segundos de la Figura 7 que el valor del PWM comandado a cada motor cambia sustancialmente, con el motor 5 (el opuesto al motor 2 en falla) casi apagado, lo cual es lógico desde el punto de vista de mantener la simetría. Además, los otros 4 motores aumentan considerablemente su empuje para compensar el bajo empuje vertical provisto por los motores 2 y 5 . El video de un vuelo realizado con este vehículo puede verse en [11].

\section{Discusión sore los resultados experimentales}

Como se mencionó anteriormente, este trabajo es el primer reporte que valida experimentalmente los resultados teóricos propuestos en [3]. Es una mejora significativa respecto a los resultados reportados en [8], dado que aquí se muestra como un hexa-rotor es capaz de volar sin perder grados de control, aún con una falla total de uno de sus motores, a diferencia de [8] en donde la falla es parcial. Debido a que luego de una falla, los motores se ven muy exigidos (para poder soportar el peso del vehículo), estos deben trabajar en un rango cercano al 60 \% del empuje total. Esto dificulta la implementación debido a que se degrada la hipótesis que la señal de PWM se relaciona linealmente con la fuerza ejercida por el rotor. Es por esto, que para lograr que el vehículo vuele con una falla total debió caracterizarse la curva que relaciona la señal de PWM comandada respecto de la fuerza y torque ejercidos por el motor. 


\section{Vehicle orientation}
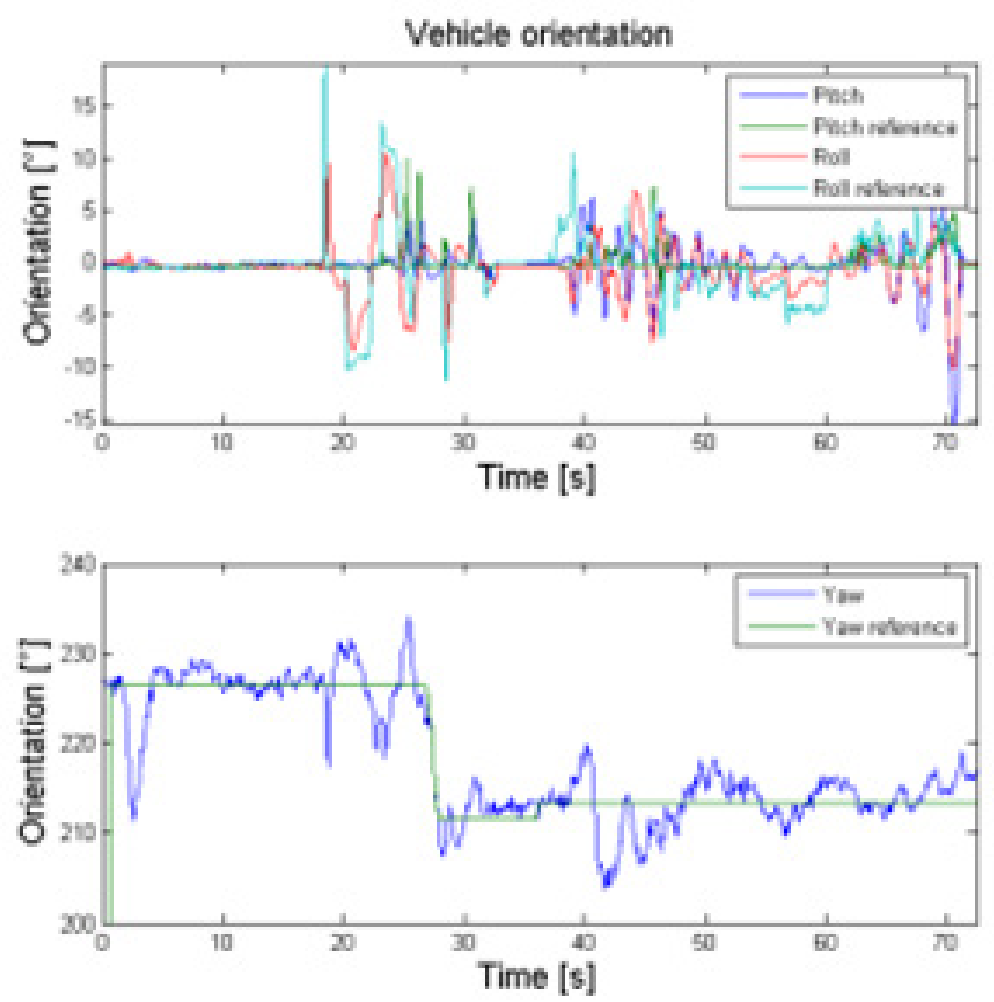

Figura 6. Orientación del vehiculo durante un vuelo con falla total

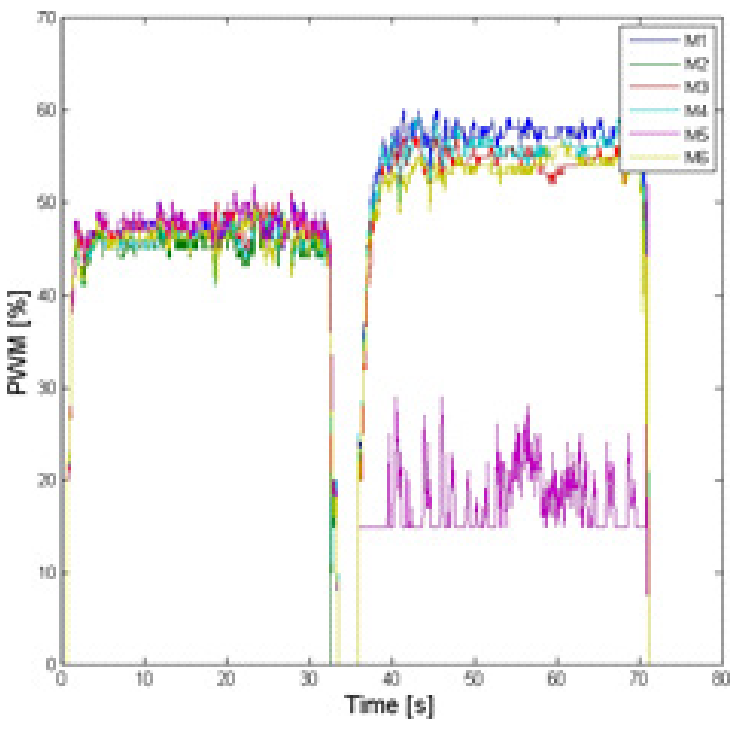

Figura 7. Valores de los PWM del vehículo durante un vuelo con falla total 
Por otro lado, debido a que los ensayos se realizaron adaptando un prototipo comercial, que no estaba pensado para volar con cinco rotores, como se mencionó anteriormente resulto necesario exigir a los motores luego de presentarse la falla, dejando poco margen para realizar maniobras. Si se quisiera un vehículo que tenga la capacidad de tolerar fallas volando en condiciones adversas y que presente cierta robustez ante perturbaciones mayores, debe pensarse en utilizar motores más potentes. Debe destacarse que en este caso se estarían sobredimensionando los motores en condiciones nominales, pero si esto implica ganar en confiabilidad y evitar posibles accidentes, es una opción que conviene considerar.

De los ensayos realizados se pueden caracterizar ciertos factores importantes que permitirán mejorar el desempeño del vehículo volando con una falla; más precisamente, permitirían incrementar la capacidad de realizar torque en la direcci6n de peor caso. El factor principal es la relación entre kt y kf, o de manera equivalente, el parámetro a. Incrementando la relación entre kt y $\mathrm{kf}$ se podría mejorar la capacidad de maniobra del vehículo ante la presencia de una falla. Esto puede analizarse estudiando la estructura de la matriz (5).

\section{CONCLUSIONES}

En este trabajo se estudia la configuraci6n de rotores in-clinados para hexa-rotores que permiten tolerar fallas totales de uno de sus rotores independientemente de cual sea el rotor en falla. Este trabajo describe los fundamentos que permiten que el vehículo pueda lograr torques en cualquier direcci6n y empuje para mantenerse en vuelo y presenta nuevos resultados experimentales. Estos resultados verifican que un hexa-rotor con los rotores inclinados puede mantenerse en vuelo en el caso de una falla total de un rotor, donde el empuje de dicho motor es nulo. Los experimentos realizados muestran un buen desempeño del sistema, en los cuales se analizan los comportamientos debido al control utilizado y a los efectos aerodinámicos como consecuencia de la asimetría de los actuadores. 


\section{REFERENCIAS}

[1] Rajappa, S., Ryll, M., Bulthoff, H. H., and Franchi, A., Modeling, Control and Design Optimization for a Fully-actuated Hexarotor Aerial Vehicle with Tilted Propellers. Proc. IEEE International Conference on Robotics and Automation (ICRA). Seattle, Washington, 2015, pp. 4006-4013.

[2] Ryll, M., Bicego, D., and Franchi, A., Modeling and Control of FAST-Hex : a Fully Actuated by Synchronized - Tilting Hexarotor. Proc. IEEE/RSJ International Conference on Intelligent Robots and Systems (IROS), 2016, pp. 1689-1694.

[3] Giribet, J. I., Sanchez-Pena, R., and Ghersin, A., Analysis and design of a tilted rotor hexacopter for fault tolerance. IEEE - Trans. on Aerospace and Electronics Systems, 4, 52, 2016.

[4] Mueller, M. W., and D'Andrea, R., Stability and control of a quadro-copter despite the complete loss of one, two, or three propellers, Proc. IEEE International Conference on Robotics and Automation (ICRA), Hong Kong, China, 2014, pp. 45-52.

[5] Achtelik, M., Doth, K.-M., Gurdan, D. and Stumpf, J., Design of a multi rotor MAV with regard to efficiency, dynamics and redundancy, Proc. AIAA Guidance, Navigation, and Control Conference, 2012.

[6] Michieletto, G., Ryll, M., and Franchi, A., Control of Statically Ho-verable MultiRotor Aerial Vehicles and Application to Rotor-Failure Robustness for Hexarotors. Proc. IEEE Int. Conf. on Robotics and Automation (ICRA), Singapore, 2017.

[7] Pose, C., Giribet, J. I., and Ghersin, A., Hexacopter fault tolerant actua-tor allocation analysis for optimal thrust. In International Conference on Unmanned Aircraft Systems (ICUAS), Miami, USA, 2017, pp. 663-671.

[8] Giribet, J. I., Pose, C. D., and Mas, I., Fault tolerant control of an hexacopter with a tilted-rotor configuration. Actas de la XVII Reuni6n de Procesamiento de la Informacion y Control. Mar del Plata, Bs. As., 2017.

[9] G. Strang. Introduction to Linear Algebra. Wellesley-Cambridge Press.

[10] Grupo de Procesamiento de Sefiales, Identificacion y Control. http://psic.fi.uba.ar/

[11] https://youtu.be/iY64D7inSfw 